\title{
On a New Intuitionistic Fuzzy Implication
}

\author{
Krassimir T. Atanassov ${ }^{1,2}$ \\ ${ }^{1}$ Department of Bioinformatics and Mathematical Modelling \\ Institute of Biophysics and Biomedical Engineering \\ Bulgarian Academy of Sciences \\ Acad. G. Bonchev Str., Bl. 105, Sofia-1113, Bulgaria \\ e-mail: krat@bas.bg \\ ${ }^{2}$ Intelligent Systems Laboratory \\ Prof. Asen Zlatarov University \\ Bourgas-8000, Bulgaria
}

\begin{abstract}
Here a new intuitionistic fuzzy implication is constructed. Its relations with some forms of Klir and Yuan's axioms are studied. Some open problems, related to the operations of intuitionistic fuzzy propositional calculus, are formulated.
\end{abstract}

Keywords: Implication, Intuitionistic fuzzy logic, Intuitionistic logic.

\section{Introduction}

The concept of "intuitionistic fuzzy propositional calculus" has been introduced about 20 years ago (see, e.g., [1, 2]). Initially, it contained only one form of conjunction, disjunction and two forms of implication. In a series of papers, [3-10], other forms of these three operations were defined. Now, there are 3 forms of the operations "conjunction" and "disjunction", 153 forms of the operation "implication" and 45 forms of the operation "negation".

Here, we introduce a new operation "implication" and study some of its properties.

In intuitionistic fuzzy propositional calculus, if $x$ is a variable, then its truth-value is represented by the ordered couple $V(x)=\langle a, b\rangle$, so that $a, b, a+b \in$ $[0,1]$, where $a$ and $b$ are degrees of validity and of non-validity of $x$.

Below, we shall assume that for the three variables $x, y$ and $z$ the following equalities hold: $V(x)=\langle a, b\rangle, V(y)=\langle c, d\rangle, V(z)=\langle e, f\rangle$, where $a, b, c, d, e, f, a+b, c+d, e+f \in[0,1]$.

For the needs of the discussion below we shall define the notion of Intuitionistic Fuzzy Tautology (IFT, see [1]) by:

$x$ is an IFT iff for $V(x)=\langle a, b\rangle$ holds: $a \geq b$,

while

$x$ is a (classical) tautology iff $a=1$ and $b=0$.

As in the case of ordinary logics, $x$ is a tautology, if $V(x)=\langle 1,0\rangle$.

\section{Main Results}

We shall start with the definition of the new implication.

$$
\begin{gathered}
V(x \rightarrow y)=\langle a, b\rangle \rightarrow\langle c, d\rangle \\
=\langle\overline{\operatorname{sg}}(\max (a, d)-\max (b, c)), \operatorname{sg}(\max (a, d)-\max (b, c))\rangle,
\end{gathered}
$$

where we use functions $\mathrm{sg}$ and $\overline{\mathrm{sg}}$ defined in the following manner:

$$
\begin{aligned}
& \operatorname{sg}(x)=\left\{\begin{array}{ll}
1 & \text { if } x>0 \\
0 & \text { if } x \leq 0
\end{array},\right. \\
& \overline{\operatorname{sg}}(x)= \begin{cases}0 & \text { if } x>0 \\
1 & \text { if } x \leq 0\end{cases}
\end{aligned}
$$

2.1. First, we see that

$$
\begin{gathered}
\overline{\operatorname{sg}}(\max (a, d)-\max (b, c))+\operatorname{sg}(\max (a, d)-\max (b, c)) \\
=1(\leq 1),
\end{gathered}
$$

i.e., the definition is correct, because for every real number $n$ : $\overline{\operatorname{sg}}(n)+\operatorname{sg}(n)=1$.

2.2. Having in mind that in the classical logic negation is obtained by the formula

$$
\neg x=x \rightarrow \overline{0},
$$

we obtain the negation, related to the new implication:

$$
\begin{gathered}
\neg\langle a, b\rangle=\langle a, b\rangle \rightarrow\langle 0,1\rangle \\
=\langle\overline{\operatorname{sg}}(\max (a, 1)-\max (b, 0)), \\
\operatorname{sg}(\max (a, 1)-\max (b, 0))\rangle \\
=\langle\overline{\operatorname{sg}}(1-b), \operatorname{sg}(1-b)\rangle .
\end{gathered}
$$

Therefore, the so generated negation coincides with negation $\neg$, e.g., in [4]. For it we directly see that it satisfies the first and the third of the following three properties and does not satisfy the second one:

Property P1: $A \rightarrow \neg \neg A$ is a tautology (an IFT), Property P2: $\neg \neg A \rightarrow A$ is a tautology (an IFT), Property P3: $\neg \neg \neg A=\neg A$. 
Really, let

$$
\begin{gathered}
X \equiv\langle a, b\rangle \rightarrow \neg \neg\langle a, b\rangle \\
=\langle a, b\rangle \rightarrow\langle\overline{\operatorname{sg}}(1-\operatorname{sg}(1-b)), \\
\operatorname{sg}(1-\operatorname{sg}(1-b))\rangle \\
=\langle\overline{\operatorname{sg}}(\max (a, \operatorname{sg}(1-\operatorname{sg}(1-b))) \\
-\max (b, \overline{\operatorname{sg}}(1-\operatorname{sg}(1-b)))), \\
\operatorname{sg}(\max (a, \operatorname{sg}(1-\operatorname{sg}(1-b))) \\
-\max (b, \overline{\operatorname{sg}}(1-\operatorname{sg}(1-b))))\rangle .
\end{gathered}
$$

If $b=1$, then $a=0$ and

$$
\begin{gathered}
X=\langle\overline{\operatorname{sg}}(\max (a, 1)-\max (1,0)), \\
\operatorname{sg}(\max (a, 1)-\max (1,0))\rangle \\
=\langle\overline{\operatorname{sg}}(1-1), \operatorname{sg}(1-1)\rangle \\
=\langle\overline{\operatorname{sg}}(0), \operatorname{sg}(0)\rangle=\langle 1,0\rangle .
\end{gathered}
$$

If $b<1$, then

$$
\begin{gathered}
X=\langle\overline{\operatorname{sg}}(\max (a, 0)-\max (b, 1)), \\
\operatorname{sg}(\max (a, 0)-\max (b, 1))\rangle \\
=\langle\overline{\operatorname{sg}}(a-1), \operatorname{sg}(a-1)\rangle=\langle 1,0\rangle .
\end{gathered}
$$

Therefore, in both cases Property P1 is satisfied as a classical tautology (and obviously, as an IFT, too).

$$
\begin{aligned}
& \text { Let } \\
& \begin{aligned}
Y \equiv \neg \neg\langle a, b\rangle \rightarrow\langle a, b\rangle \\
=\langle\overline{\operatorname{sg}}(1-\operatorname{sg}(1-b)), \operatorname{sg}(1-\operatorname{sg}(1-b)))\rangle \rightarrow\langle a, b\rangle \\
=\langle\overline{\operatorname{sg}}(\max (\overline{\operatorname{sg}}(1-\operatorname{sg}(1-b)), b) \\
-\max (\operatorname{sg}(1-\operatorname{sg}(1-b)), a)), \\
\operatorname{sg}(\max (\overline{\operatorname{sg}}(1-\operatorname{sg}(1-b)), b) \\
-\max (\operatorname{sg}(1-\operatorname{sg}(1-b)), a))\rangle .
\end{aligned}
\end{aligned}
$$

If $b=1$, then $a=0$ and

$$
\begin{gathered}
Y=\langle\overline{\mathrm{sg}}(\max (0,1)-\max (1,0)), \operatorname{sg}(\max (0,1) \\
-\max (1,0))\rangle=\langle 1,0\rangle,
\end{gathered}
$$

while, if $b<1$ we obtain

$$
\begin{gathered}
Y=\langle\overline{\operatorname{sg}}(\max (1, b)-\max (0, a)), \\
\operatorname{sg}(\max (1, b)-\max (0, a))\rangle \\
=\langle\overline{\operatorname{sg}}(1-a), \operatorname{sg}(1-a)\rangle .
\end{gathered}
$$

If $a=1$, then $b=0$ and

$$
Y=\langle\overline{\operatorname{sg}}(1-1), \operatorname{sg}(1-1)\rangle=\langle 1,0\rangle
$$

but if $a<1$, then

$$
Y=\langle 0,1\rangle
$$

i.e., $Y$ is not a tautology, and it is not an IFT, either.
For the Property P3 we directly obtain that

$$
\begin{gathered}
\neg \neg \neg\langle a, b\rangle \rightarrow \neg\langle a, b\rangle \\
=\neg \neg\langle\overline{\operatorname{sg}}(1-b), \operatorname{sg}(1-b)\rangle \\
\rightarrow\langle\overline{\operatorname{sg}}(1-b), \operatorname{sg}(1-b)\rangle \\
=\neg\langle\overline{\operatorname{sg}}(1-\operatorname{sg}(1-b)), \operatorname{sg}(1-\operatorname{sg}(1-b)) \\
\rangle \rightarrow\langle\overline{\operatorname{sg}}(1-b), \operatorname{sg}(1-b)\rangle \\
=\neg\langle\overline{\operatorname{sg}}(1-b), \operatorname{sg}(1-b)\rangle \rightarrow\langle\overline{\operatorname{sg}}(1-b), \operatorname{sg}(1-b)\rangle \\
=\langle 1,0\rangle,
\end{gathered}
$$

i.e., in both cases Property P3 is satisfied as a tautology (and obviously, as an IFT, too).

2.3. Some variants of fuzzy implications (marked by $I(x, y))$ are described in book [12] by Georg Klir and Bo Yuan and the following nine axioms are discussed, where

$$
I(x, y) \equiv x \rightarrow y
$$

Axiom $1(\forall x, y)(x \leq y \rightarrow(\forall z)(I(x, z) \geq I(y, z)))$.

Axiom $2(\forall x, y)(x \leq y \rightarrow(\forall z)(I(z, x) \leq I(z, y)))$.

Axiom $3(\forall y)(I(0, y)=1)$.

Axiom $4(\forall y)(I(1, y)=y)$.

Axiom $5(\forall x)(I(x, x)=1)$.

Axiom $6(\forall x, y, z)(I(x, I(y, z))=I(y, I(x, z)))$.

Axiom $7(\forall x, y)(I(x, y)=1$ iff $x \leq y)$.

Axiom $8(\forall x, y)(I(x, y)=I(N(y), N(x)))$, where $N$ is an operation for a negation.

Axiom $9 I$ is a continuous function.

Below, we study which axioms are valid for the new implication and in which form (as classical tautologies or as IFTs).

Let $a \leq c$ and $b \geq d$. Then for Axiom 1 we obtain:

$$
\begin{gathered}
V(x \rightarrow z)=\langle\overline{\operatorname{sg}}(\max (a, f)-\max (b, e)), \\
\operatorname{sg}(\max (a, f)-\max (b, e))\rangle
\end{gathered}
$$

and

$$
\begin{gathered}
V(y \rightarrow z)=\langle\overline{\operatorname{sg}}(\max (c, f)-\max (d, e)), \\
\operatorname{sg}(\max (c, f)-\max (d, e))\rangle .
\end{gathered}
$$

Let

$$
\begin{gathered}
X \equiv \overline{\operatorname{sg}}(\max (a, f)-\max (b, e)) \\
-\overline{\operatorname{sg}}(\max (c, f)-\max (d, e)) .
\end{gathered}
$$

If $\max (a, f)>\max (b, e)$, then

$$
\max (c, f) \geq \max (a, f) \geq \max (b, e) \geq \max (d, e),
$$

i.e.

$$
\max (c, f) \geq \max (d, e),
$$

and

$$
X=0-0=0 .
$$

If $\max (a, f) \leq \max (b, e)$, then

$$
X=1-\overline{\operatorname{sg}}(\max (c, f)-\max (d, e)) \geq 0 .
$$


Analogously we see that

$$
\begin{gathered}
Y=\operatorname{sg}(\max (c, f)-\max (d, e)) \\
-\operatorname{sg}(\max (a, f)-\max (b, e)) \geq 0 .
\end{gathered}
$$

Hence, Axiom 1 is valid.

The validity of Axiom 2 is checked analogously.

For Axiom 3 we obtain:

$$
\begin{gathered}
\langle 0,1\rangle \rightarrow\langle c, d\rangle \\
=\langle\overline{\operatorname{sg}}(\max (0, c)-\max (1, d)), \\
\operatorname{sg}(\max (0, d)-\max (1, c))\rangle \\
=\langle\overline{\operatorname{sg}}(c-1), \operatorname{sg}(d-1)\rangle=\langle 1,0\rangle,
\end{gathered}
$$

i.e., it is valid.

Now, we must mention that Axiom 4 is not valid, because

$$
\begin{gathered}
X \equiv\langle 1,0\rangle \rightarrow\langle c, d\rangle \\
=\langle\overline{\operatorname{sg}}(\max (1, d)-\max (0, c)), \\
\operatorname{sg}(\max (1, d)-\max (0, c))\rangle \\
=\langle\overline{\operatorname{sg}}(1-c), \operatorname{sg}(1-c)\rangle .
\end{gathered}
$$

If $c=1$, then

$$
X=\langle 1,0\rangle
$$

while, if $c<1$, then

$$
X=\langle 0,1\rangle \leq\langle c, d\rangle .
$$

On the other hand

$$
\begin{gathered}
\langle 1,0\rangle \rightarrow \neg \neg\langle a, b\rangle \\
=\langle 1,0\rangle \rightarrow \neg \neg\langle\operatorname{sg}(1-b), \overline{\operatorname{sg}}(1-b)\rangle \\
=\langle\overline{\operatorname{sg}}(\max (1, \overline{\operatorname{sg}}(1-b))-\max (0, \operatorname{sg}(1-b))), \\
\operatorname{sg}(\max (1, \overline{\operatorname{sg}}(1-b) d)-\max (0, \operatorname{sg}(1-b)))\rangle . \\
=\langle\overline{\operatorname{sg}}(1-\operatorname{sg}(1-b)), \operatorname{sg}(1-\operatorname{sg}(1-b))\rangle \\
=\langle\operatorname{sg}(1-b), \overline{\operatorname{sg}}(1-b)\rangle=\neg \neg\langle a, b\rangle .
\end{gathered}
$$

Hence, we can change Axiom 4 to one of the following two forms:

Axiom 4* $(\forall y)(I(1, \neg \neg y)=\neg \neg y)$

Axiom $4^{* *}(\forall y)(I(1, y) \leq y)$

and in any of these forms the axiom will be valid. Obviously, in the classical logic, where $\neg \neg x=x$, Axiom 4* coincides with Axiom 4.

For Axiom 5 we obtain:

$$
\begin{gathered}
\langle a, b\rangle \rightarrow\langle a, b\rangle \\
=\langle\overline{\operatorname{sg}}(\max (a, b)-\max (b, a)), \\
\operatorname{sg}(\max (a, b)-\max (b, a))\rangle=\langle 1,0\rangle,
\end{gathered}
$$

i.e., it is valid.

Now, we see that Axiom 6 is not valid. Really, sequentially we see that

$$
V(x \rightarrow(y \rightarrow z))=\langle a, b\rangle \rightarrow(\langle c, d\rangle \rightarrow\langle e, f\rangle)
$$

$$
\begin{gathered}
=\langle a, b\rangle \rightarrow(\langle\overline{\operatorname{sg}}(\max (c, f)-\max (d, e)), \\
\quad \operatorname{sg}(\max (c, f)-\max (d, e))\rangle) \\
=\langle\overline{\operatorname{sg}}(\max (a, \operatorname{sg}(\max (c, f)-\max (d, e))) \\
-\max (b, \overline{\operatorname{sg}}(\max (c, f)-\max (d, e)))), \\
\operatorname{sg}(\max (a, \operatorname{sg}(\max (c, f)-\max (d, e))) \\
-\max (b, \overline{\operatorname{sg}}(\max (c, f)-\max (d, e))))\rangle
\end{gathered}
$$

and

$$
\begin{gathered}
V(y \rightarrow(x \rightarrow z))=\langle c, d\rangle \rightarrow(\langle a, b\rangle \rightarrow\langle e, f\rangle) \\
=\langle c, d\rangle \rightarrow(\langle\overline{\operatorname{sg}}(\max (a, f)-\max (b, e)) \\
\operatorname{sg}(\max (a, f)-\max (b, e))\rangle) \\
=\langle\overline{\operatorname{sg}}(\max (c, \operatorname{sg}(\max (a, f)-\max (b, e))) \\
-\max (d, \overline{\operatorname{sg}}(\max (a, f)-\max (b, e)))) \\
\operatorname{sg}(\max (c, \operatorname{sg}(\max (a, f)-\max (b, e))) \\
-\max (d, \overline{\operatorname{sg}}(\max (a, f)-\max (b, e))))\rangle
\end{gathered}
$$

Let

$$
\begin{aligned}
X & \equiv \overline{\operatorname{sg}}(\max (a, \operatorname{sg}(\max (c, f)-\max (d, e))) \\
& -\max (b, \overline{\operatorname{sg}}(\max (c, f)-\max (d, e)))) \\
& -\overline{\operatorname{sg}}(\max (c, \operatorname{sg}(\max (a, f)-\max (b, e))) \\
& -\max (d, \overline{\operatorname{sg}}(\max (a, f)-\max (b, e)))) .
\end{aligned}
$$

If $\max (c, f)>\max (d, e)$, then

$$
\begin{gathered}
X \equiv \overline{\operatorname{sg}}(\max (a, 1)-\max (b, 0)) \\
-\overline{\operatorname{sg}}(\max (c, \operatorname{sg}(\max (a, f) \\
-\max (b, e)))-\max (d, \overline{\operatorname{sg}}(\max (a, f)-\max (b, e)))) \\
=\overline{\operatorname{sg}}(1-b)-\overline{\operatorname{sg}}(\max (c, \operatorname{sg}(\max (a, f)-\max (b, e))) \\
-\max (d, \overline{\operatorname{sg}}(\max (a, f)-\max (b, e)))) .
\end{gathered}
$$

If $b=1$, then

$$
\begin{aligned}
X= & 1-\overline{\operatorname{sg}}(\max (c, \operatorname{sg}(\max (a, f)-1)) \\
& -\max (d, \overline{\operatorname{sg}}(\max (a, f)-1)))
\end{aligned}
$$$$
=1-\overline{\operatorname{sg}}(\max (c, 0)-\max (d, 0))=1-\overline{\operatorname{sg}}(c-d) .
$$

Therefore, there are cases, when $X=1$, and other cases in which $X=0$.

On the other hand, we change the form of Axiom 6 to the form

Axiom 6* $(\forall x, y, z)(I(N(N(x)), I(N(N(y))$, $N(N(z))))=I(N(N(y)), I(N(N(x)), N(N(z)))))$.

For that, we calculate

$$
\begin{gathered}
V(\neg \neg x \rightarrow(\neg \neg y \rightarrow \neg \neg z)) \\
=\neg \neg\langle a, b\rangle \rightarrow(\neg \neg\langle c, d\rangle \rightarrow \neg \neg\langle e, f\rangle) \\
=\langle\operatorname{sg}(1-b), \overline{\operatorname{sg}}(1-b)\rangle \rightarrow(\langle\operatorname{sg}(1-d), \overline{\operatorname{sg}}(1-d)\rangle \\
\rightarrow\langle\operatorname{sg}(1-f), \overline{\operatorname{sg}}(1-f)\rangle) \\
=\langle\operatorname{sg}(1-b), \overline{\operatorname{sg}}(1-b)\rangle \rightarrow\langle\overline{\operatorname{sg}}(\max (\operatorname{sg}(1-d), \overline{\operatorname{sg}}(1-f))
\end{gathered}
$$




$$
-\max (\overline{\operatorname{sg}}(1-d), \operatorname{sg}(1-f))),
$$

$\operatorname{sg}(\max (\operatorname{sg}(1-d), \overline{\operatorname{sg}}(1-f))-\max (\overline{\operatorname{sg}}(1-d), \operatorname{sg}(1-f)))\rangle$

$$
=\langle\overline{\operatorname{sg}}(\max (\operatorname{sg}(1-b), \operatorname{sg}(\max (\operatorname{sg}(1-d), \overline{\operatorname{sg}}(1-f))
$$$$
-\max (\overline{\operatorname{sg}}(1-d), \operatorname{sg}(1-f))))-\max (\overline{\operatorname{sg}}(1-b),
$$

$\overline{\operatorname{sg}}(\max (\operatorname{sg}(1-d), \overline{\operatorname{sg}}(1-f))$

$-\max (\overline{\operatorname{sg}}(1-d), \operatorname{sg}(1-f)))))$,

$\operatorname{sg}(\max (\operatorname{sg}(1-b), \operatorname{sg}(\max (\operatorname{sg}(1-d), \overline{\operatorname{sg}}(1-f))$

$-\max (\overline{\operatorname{sg}}(1-d), \operatorname{sg}(1-f))))-\max (\overline{\operatorname{sg}}(1-b)$,

$\overline{\operatorname{sg}}(\max (\operatorname{sg}(1-d), \overline{\operatorname{sg}}(1-f))$

$-\max (\overline{\operatorname{sg}}(1-d), \operatorname{sg}(1-f)))))\rangle$

and

$$
\begin{gathered}
V(\neg \neg y \rightarrow(\neg \neg x \rightarrow \neg \neg z)) \\
=\neg \neg\langle c, d\rangle \rightarrow(\neg \neg\langle a, b\rangle \rightarrow \neg \neg\langle e, f\rangle) \\
=\langle\operatorname{sg}(1-d), \overline{\operatorname{sg}}(1-d)\rangle \rightarrow(\langle\operatorname{sg}(1-b), \overline{\operatorname{sg}}(1-b)\rangle \\
\rightarrow\langle\operatorname{sg}(1-f), \overline{\operatorname{sg}}(1-f)\rangle) \\
=\langle\operatorname{sg}(1-d), \overline{\operatorname{sg}}(1-d)\rangle \rightarrow\langle\overline{\operatorname{sg}}(\max (\operatorname{sg}(1-b), \overline{\operatorname{sg}}(1-f)) \\
-\max (\overline{\operatorname{sg}}(1-b), \operatorname{sg}(1-f))),
\end{gathered}
$$

$\operatorname{sg}(\max (\operatorname{sg}(1-b), \overline{\operatorname{sg}}(1-f))-\max (\overline{\operatorname{sg}}(1-b), \operatorname{sg}(1-f)))\rangle$.

$=\langle\overline{\operatorname{sg}}(\max (\operatorname{sg}(1-d), \operatorname{sg}(\max (\operatorname{sg}(1-b), \overline{\operatorname{sg}}(1-f))$

$-\max (\overline{\operatorname{sg}}(1-b), \operatorname{sg}(1-f))))-\max (\overline{\operatorname{sg}}(1-d)$,

$\overline{\operatorname{sg}}(\max (\operatorname{sg}(1-b), \overline{\operatorname{sg}}(1-f))-\max (\overline{\operatorname{sg}}(1-b), \operatorname{sg}(1-f)))))$,

$\operatorname{sg}(\max (\operatorname{sg}(1-d), \operatorname{sg}(\max (\operatorname{sg}(1-b), \overline{\operatorname{sg}}(1-f))$

$-\max (\overline{\operatorname{sg}}(1-b), \operatorname{sg}(1-f))))-\max (\overline{\operatorname{sg}}(1-d)$,

$\overline{\mathrm{sg}}(\max (\operatorname{sg}(1-b), \overline{\mathrm{sg}}(1-f))$

$-\max (\overline{\operatorname{sg}}(1-b), \operatorname{sg}(1-f)))))\rangle$.

Let

$X \equiv \overline{\operatorname{sg}}(\max (\operatorname{sg}(1-b), \operatorname{sg}(\max (\operatorname{sg}(1-d), \overline{\operatorname{sg}}(1-f))$

$-\max (\overline{\operatorname{sg}}(1-d), \operatorname{sg}(1-f))))-\max (\overline{\operatorname{sg}}(1-b)$,

$\overline{\operatorname{sg}}(\max (\operatorname{sg}(1-d), \overline{\operatorname{sg}}(1-f))-\max (\overline{\operatorname{sg}}(1-d), \operatorname{sg}(1-f)))))$

$-\overline{\operatorname{sg}}(\max (\operatorname{sg}(1-d), \operatorname{sg}(\max (\operatorname{sg}(1-b), \overline{\operatorname{sg}}(1-f))$

$-\max (\overline{\operatorname{sg}}(1-b), \operatorname{sg}(1-f))))-\max (\overline{\operatorname{sg}}(1-d)$,

$\overline{\operatorname{sg}}(\max (\operatorname{sg}(1-b), \overline{\operatorname{sg}}(1-f))$

$-\max (\overline{\operatorname{sg}}(1-b), \operatorname{sg}(1-f)))))$.

If $b=1$, then

$$
\begin{gathered}
X=\overline{\operatorname{sg}}(\max (0, \operatorname{sg}(\max (\operatorname{sg}(1-d), \overline{\operatorname{sg}}(1-f)) \\
-\max (\overline{\operatorname{sg}}(1-d), \operatorname{sg}(1-f))))-\max (1,
\end{gathered}
$$

$\overline{\operatorname{sg}}(\max (\operatorname{sg}(1-d), \overline{\operatorname{sg}}(1-f))-\max (\overline{\operatorname{sg}}(1-d), \operatorname{sg}(1-f)))))$

$-\overline{\operatorname{sg}}(\max (\operatorname{sg}(1-d), \operatorname{sg}(\max (0, \overline{\operatorname{sg}}(1-f))$

$-\max (1, \operatorname{sg}(1-f))))-\max (\overline{\operatorname{sg}}(1-d)$,

$\overline{\operatorname{sg}}(\max (0, \overline{\operatorname{sg}}(1-f))-\max (1, \operatorname{sg}(1-f)))))$

$=\overline{\operatorname{sg}}(\operatorname{sg}(\max (\operatorname{sg}(1-d), \overline{\operatorname{sg}}(1-f))$

$$
-\max (\overline{\operatorname{sg}}(1-d), \operatorname{sg}(1-f)))-1),
$$

$-\overline{\operatorname{sg}}(\max (\operatorname{sg}(1-d), \operatorname{sg}(\max (0, \overline{\operatorname{sg}}(1-f))-1))$

$-\max (\overline{\operatorname{sg}}(1-d), \overline{\operatorname{sg}}(\overline{\operatorname{sg}}(1-f)-1)))$

$=1-\overline{\operatorname{sg}}(\max (\operatorname{sg}(1-d), \operatorname{sg}(\overline{\operatorname{sg}}(1-f)-1))$

$$
-\max (\overline{\operatorname{sg}}(1-d), 1))
$$

$=1-\overline{\operatorname{sg}}(\max (\operatorname{sg}(1-d), 0)-1)=1-1=0$.

If $b<1$, then

$$
\begin{gathered}
X=\overline{\operatorname{sg}}(\max (1, \operatorname{sg}(\max (\operatorname{sg}(1-d), \overline{\operatorname{sg}}(1-f)) \\
-\max (\overline{\operatorname{sg}}(1-d), \operatorname{sg}(1-f))))-\max (0, \\
\quad \overline{\operatorname{sg}}(\max (\operatorname{sg}(1-d), \overline{\operatorname{sg}}(1-f)) \\
-\max (\overline{\operatorname{sg}}(1-d), \operatorname{sg}(1-f)))))
\end{gathered}
$$

$-\overline{\operatorname{sg}}(\max (\operatorname{sg}(1-d), \operatorname{sg}(\max (1, \overline{\operatorname{sg}}(1-f))$

$-\max (0, \operatorname{sg}(1-f))))-\max (\overline{\operatorname{sg}}(1-d), \overline{\operatorname{sg}}(\max (1$,

$$
\overline{\operatorname{sg}}(1-f))-\max (0, \operatorname{sg}(1-f))))
$$

$=\overline{\operatorname{sg}}(1-\overline{\operatorname{sg}}(\max (\operatorname{sg}(1-d), \overline{\operatorname{sg}}(1-f))$

$-\max (\overline{\operatorname{sg}}(1-d), \operatorname{sg}(1-f))))-\overline{\operatorname{sg}}(\max (\operatorname{sg}(1-d)$, $\operatorname{sg}(1-\operatorname{sg}(1-f)))-\max (\overline{\operatorname{sg}}(1-d), \overline{\operatorname{sg}}(1-\operatorname{sg}(1-f))))$.

If $d=1$, then

$X=\overline{\operatorname{sg}}(1-\overline{\operatorname{sg}}(\max (0, \overline{\operatorname{sg}}(1-f))-\max (1, \operatorname{sg}(1-f))))$

$-\overline{\operatorname{sg}}(\max (0, \operatorname{sg}(1-\operatorname{sg}(1-f)))$

$-\max (1, \overline{\operatorname{sg}}(1-\operatorname{sg}(1-f))))$

$=\overline{\operatorname{sg}}(1-\overline{\operatorname{sg}}(\overline{\operatorname{sg}}(1-f)-1))-\overline{\operatorname{sg}}(\operatorname{sg}(1-\operatorname{sg}(1-f))-1)$

$=\overline{\operatorname{sg}}(1-1)-1=1-1=0$.

If $d<1$, then

$X=\overline{\operatorname{sg}}(1-\overline{\operatorname{sg}}(\max (1, \overline{\operatorname{sg}}(1-f))-\max (0, \operatorname{sg}(1-f))))$

$$
\begin{aligned}
& -\overline{\operatorname{sg}}(\max (1, \operatorname{sg}(1-\operatorname{sg}(1-f))) \\
& -\max (0, \overline{\operatorname{sg}}(1-\operatorname{sg}(1-f))))
\end{aligned}
$$

$=\overline{\operatorname{sg}}(1-\overline{\operatorname{sg}}(1-\operatorname{sg}(1-f)))-\overline{\operatorname{sg}}(1-\overline{\operatorname{sg}}(1-\operatorname{sg}(1-f)))=0$.

Therefore, the new implication satisfies Axiom $6^{*}$.

For the check of Axiom 7, we first see that if $\langle a, b\rangle \leq\langle c, d\rangle$, i.e., $a \leq c$ and $d \leq b$, then

$$
\langle a, b\rangle \rightarrow\langle c, d\rangle
$$

$$
=\langle\overline{\operatorname{sg}}(\max (a, d)-\max (b, c)),
$$

$\operatorname{sg}(\max (a, d)-\max (b, c))\rangle=\langle 1,0\rangle$.

The opposite direction is not valid, because if

$$
\begin{gathered}
\langle a, b\rangle \rightarrow\langle c, d\rangle \\
=\langle\overline{\operatorname{sg}}(\max (a, d)-\max (b, c)),
\end{gathered}
$$

$\operatorname{sg}(\max (a, d)-\max (b, c))\rangle=\langle 1,0\rangle$.

then

$\overline{\operatorname{sg}}(\max (a, d)-\max (b, c))=1$, 


$$
\operatorname{sg}(\max (a, d)-\max (b, c))=0,
$$

i.e. $\max (a, d) \leq \max (b, c)$, but from here it does not follow that $\langle a, b\rangle \leq\langle c, d\rangle$.

Therefore, Axiom 7 is valid in the form:

Axiom $7^{*}(\forall x, y)$ (if $x \leq y$, then $\left.I(x, y)\right)$.

For Axiom 8, as above, we see that it is not valid in the original form. Now, we check its new form

Axiom 8* $(\forall x, y)(N(N(I(N(N(x)), N(N(y)))))$ $=I(N(y), N(x)))$.

Now, we calculate sequentially:

$$
\begin{gathered}
V(\neg \neg(\neg \neg x \rightarrow \neg \neg y)) \\
=\neg \neg(\neg \neg\langle a, b\rangle \rightarrow \neg \neg\langle c, d\rangle) \\
=\neg \neg(\langle\operatorname{sg}(1-b), \overline{\operatorname{sg}}(1-b)\rangle \\
\rightarrow \neg \neg\langle\operatorname{sg}(1-d), \overline{\operatorname{sg}}(1-d)\rangle) \\
=\neg \neg\langle\overline{\operatorname{sg}}(\max (\operatorname{sg}(1-b), \\
\overline{\operatorname{sg}}(1-d))-\max (\overline{\operatorname{sg}}(1-b), \operatorname{sg}(1-d))), \\
\operatorname{sg}(\max (\operatorname{sg}(1-b), \overline{\operatorname{sg}}(1-d)) \\
-\max (\overline{\operatorname{sg}}(1-b), \operatorname{sg}(1-d)))\rangle \\
\langle\operatorname{sg}(1-\operatorname{sg}(\max (\operatorname{sg}(1-b), \overline{\operatorname{sg}}(1-d)) \\
-\max (\overline{\operatorname{sg}}(1-b), \operatorname{sg}(1-d)))), \\
\overline{\operatorname{sg}}(1-\operatorname{sg}(\max (\operatorname{sg}(1-b), \overline{\operatorname{sg}}(1-d)) \\
-\max (\overline{\operatorname{sg}}(1-b), \operatorname{sg}(1-d)))) .
\end{gathered}
$$

and

$$
\begin{gathered}
V(\neg y \rightarrow \neg x)=\neg\langle c, d\rangle \rightarrow \neg\langle a, b\rangle \\
=\langle\overline{\operatorname{sg}}(1-d), \operatorname{sg}(1-d)\rangle \\
\rightarrow\langle\overline{\operatorname{sg}}(1-b), \operatorname{sg}(1-b)\rangle \\
=\langle\overline{\operatorname{sg}}(\max (\overline{\operatorname{sg}}(1-d), \operatorname{sg}(1-b)) \\
-\max (\operatorname{sg}(1-d), \overline{\operatorname{sg}}(1-b))), \\
\operatorname{sg}(\max (\overline{\operatorname{sg}}(1-d), \operatorname{sg}(1-b)) \\
-\max (\operatorname{sg}(1-d), \overline{\operatorname{sg}}(1-b)))\rangle .
\end{gathered}
$$

Let

$$
\begin{aligned}
X \equiv & \operatorname{sg}(1-\operatorname{sg}(\max (\operatorname{sg}(1-b), \overline{\operatorname{sg}}(1-d)) \\
& -\max (\overline{\operatorname{sg}}(1-b), \operatorname{sg}(1-d)))) \\
& -\overline{\operatorname{sg}}(\max (\overline{\operatorname{sg}}(1-d), \operatorname{sg}(1-b)) \\
& -\max (\operatorname{sg}(1-d), \overline{\operatorname{sg}}(1-b))) .
\end{aligned}
$$

If $b=1$, then

$$
\begin{gathered}
X=\operatorname{sg}(1-\operatorname{sg}(\max (0, \overline{\operatorname{sg}}(1-d))-\max (1, \operatorname{sg}(1-d)))) \\
-\overline{\operatorname{sg}}(\max (\overline{\operatorname{sg}}(1-d), 0)-\max (\operatorname{sg}(1-d), 1)) \\
=\operatorname{sg}(1-\operatorname{sg}(\overline{\operatorname{sg}}(1-d)-1))-\overline{\operatorname{sg}}(\overline{\operatorname{sg}}(1-d)-1) \\
=\operatorname{sg}(1)-\overline{\operatorname{sg}}(0)=0 .
\end{gathered}
$$

If $b<1$, then

$$
\begin{aligned}
X= & \operatorname{sg}(1-\operatorname{sg}(\max (1, \overline{\operatorname{sg}}(1-d))-\max (0, \operatorname{sg}(1-d)))) \\
& -\overline{\operatorname{sg}}(\max (\overline{\operatorname{sg}}(1-d), 1)-\max (\operatorname{sg}(1-d), 0))
\end{aligned}
$$

$=\operatorname{sg}(1-\operatorname{sg}(1-\operatorname{sg}(1-d)))-\overline{\operatorname{sg}}(1-\operatorname{sg}(1-d))$.

If $d=1$, then

$$
X=\operatorname{sg}(1-\operatorname{sg}(1))-\overline{\operatorname{sg}}(1-0)=0-0=0 .
$$

If $d<1$, then

$$
X=\operatorname{sg}(1-\operatorname{sg}(1-1))-\overline{\operatorname{sg}}(1-1)=1-1=0 .
$$

Therefore, the first members of the two expressions coincide. Analogously, we see that the second members of the two expressions coincide, as well.

Obviously, the existence of functions sg and $\overline{\mathrm{sg}}$ in the definition of the new operation ignores the possibility for the validity of Axiom 9.

Finally, on the basis of the above discussion, we can formulate the following:

Theorem. The new implication satisfies Axioms 1, 2, 3, $4^{*}$ (and $4^{* *}$ ), 5, 6*, $7^{*}, 8^{*}$, as tautologies (and therefore, as IFTs).

\section{Conclusion}

In the next step of this research, other new implications will be introduced and studied. All they show that intuitionistic fuzzy sets and logics in the sense, described in $[2,4]$ correspond to the ideas of Brouwer's intuitionism.

It is worth noting that the search of new implications and negations is important for constructing of rules in various decision making, decision support and Intercriteria analysis applications of intuitionistic fuzzy sets.

Also, these are needed for developing of a new version of the Intuitionistic Fuzzy Prolog, like the one described in [5].

\section{Acknowledgements}

The author is thankful for the support provided by the Bulgarian National Science Fund under Grant Ref. No. DFNI-I-02-5.

\section{References}

[1] K. Atanassov, Two variants of intuitonistc fuzzy propositional calculus. Preprint IM-MFAIS-588, Sofia, 1988.

[2] K. Atanassov, Intuitionistic Fuzzy Sets. Springer, Heidelberg, 1999.

[3] K. Atanassov, Remarks on the conjunctions, disjunctions and implications of the intuitionistic fuzzy logic. International Journal of Uncertainty, Fuzziness and Knowledge-Based Systems, 9(1):55-65, 2001.

[4] K. Atanassov, On Intuitionistic Fuzzy Sets. Springer, Berlin, 2012.

[5] K. Atanassov, and C. Georgiev, Intuitionistic fuzzy Prolog, Fuzzy Sets and Systems, 53(1):121-128, 1993. 
[6] L. Atanassova, A new intuitionistic fuzzy implication, Cybernetics and Information Technologies, 9(2):21-25, 2009.

[7] L. Atanassova, On two modifications of the intuitionistic fuzzy implication $\rightarrow @$, Notes on Intuitionistic Fuzzy Sets, 18(2):26-30, 2012.

[8] L. Atanassova, On the modal form of the intuitionistic fuzzy implications $\rightarrow_{@}^{\prime}$ and $\rightarrow \rightarrow_{@}^{\prime \prime}, I s-$ sues in Intuitionistic Fuzzy Sets and Generalized Nets, 10:5-11, 2013.

[9] P. Dworniczak, Some remarks about the L. Atanassova's paper "A new intuitionistic fuzzy implication", Cybernetics and Information Technologies, 10(3):3-9, 2010.

[10] P. Dworniczak, On one class of intuitionistic fuzzy implications, Cybernetics and Information Technologies, 10(4):13-21, 2010.

[11] P. Dworniczak, On some two-parametric intuitionistic fuzzy implication, Notes on Intuitionistic Fuzzy Sets, 17(2):8-16, 2011.

[12] G. Klir, and B. Yuan, Fuzzy Sets and Fuzzy Logic. Prentice Hall, New Jersey, 1995. 